\title{
C-reactive protein and procalcitonin as predictors of survival and septic shock in ventilator-associated pneumonia
}

\author{
G. Hillas*, T. Vassilakopoulos” , P. Plantza*, A. Rasidakis* and P. Bakakos*
}

ABSTRACT: We evaluated the performance of procalcitonin (PCT) and C-reactive protein (CRP) threshold values and kinetics as predictors of ventilator-associated pneumonia (VAP) survival and septic shock development.

45 adult patients with VAP were studied. Serum CRP and PCT levels and the Sequential Organ Failure Assessment (SOFA) score were measured on days 1, 4 and 7 (D1, D4, D7) of VAP and their variations between different days (kinetics) were calculated ( $\triangle P C T, \triangle C R P$ ). A multivariate logistic regression model was constructed with either VAP 28-day survival or septic shock development as dependent variables, and PCT values, CRP values, kinetics, age, sex, SOFA and Acute Physiology and Chronic Health Evaluation (APACHE) II score as independent variables.

No difference was found in CRP levels between survivors and nonsurvivors. Nonsurvivors had significantly higher PCT levels on D1 and D7. In the multivariate analysis, the only factors predicting VAP survival were $\triangle \mathrm{PCT}_{7-1}$ (OR 7.23, 95\% Cl 0.008-0.468) and $\Delta \mathrm{CRP}_{7-4}(\mathrm{OR} 4.59,95 \%$ $\mathrm{Cl}$ 0.013-0.824). VAP patients who developed septic shock had significantly higher CRP levels on D1 and D7 and higher PCT levels on D1 and D4. The only factor predicting the development of septic shock was SOFA on D1 (OR 7.44, 95\% CI 1.330-5.715).

Neither PCT and CRP threshold values nor their kinetics can predict VAP survival or septic shock development.

KEYWORDS: C-reactive protein, outcome, procalcitonin, septic shock, Sequential Organ Failure Assessment score, ventilator-associated pneumonia

$\mathbf{V}$ entilator-associated pneumonia (VAP) is the most common acquired infection among intensive care unit (ICU) patients $[1,2]$. The risk for VAP rises $1-3 \%$ for each day the patient remains on mechanical ventilation [3].

VAP is a common problem in ICUs and its clinical and microbiological diagnoses, risk factors, preventive measures and empiric therapy are still under consideration by specialists. VAP prolongs the length of ICU stay and is associated with a $20-30 \%$ increase in the risk of death $[4,5]$. The mortality rate for VAP ranges $24-50 \%$ and can reach $76 \%$ in specific settings or when lung infection is caused by multidrug-resistant pathogens $[6,7]$.

The evaluation of the response of VAP to antibiotic treatment relies on a combination of clinical, radiological and bacteriological criteria. To date, no single clinical or biological indicator

For editorial comments see page 725 . has gained unanimous acceptance in the assessment of the response to treatment, although several attempts have been made to correlate them with the prognosis of VAP $[8,9]$. Many markers have been proposed to be the most promising candidates, such as leukocyte count, C-reactive protein (CRP) and procalcitonin (PCT). $\mathrm{CRP}$ is an acute-phase protein produced by the liver. PCT is the prehormone of calcitonin that is normally secreted by the C-cells of the thyroid in response to hypercalcaemia. Under normal conditions, negligible serum PCT levels are detected. In systemic infections, sepsis and sepsis-like conditions, serum levels of PCT are markedly elevated, although its origin remains a matter of discussion. The levels of serum PCT correlate positively with the severity of the illness and mortality [10]. LUYT et al. [11] suggested that serum procalcitonin levels $>1 \mathrm{ng} \cdot \mathrm{mL}^{-1}$ on the first day, $>1.5 \mathrm{ng} \cdot \mathrm{mL}^{-1}$ on the third day and $>0.5 \mathrm{ng} \cdot \mathrm{mL}^{-1}$ on the seventh day in patients with VAP were strong predictors of unfavourable outcome, defined as either death, recurrent VAP

\section{AFFILIATIONS}

*Dept of Respiratory and Critical Care Medicine, "Sotiria" Chest Diseases Hospital, and

\#Dept of Critical Care and Pulmonary Services, University of Athens Medical School, Evangelismos Hospital, Athens, Greece

CORRESPONDENCE

P. Bakakos

11 Kononos St

Pagrati

Athens

Greece

E-mail: petros44@hotmail.com

Received:

March 292009

Accepted after revision:

Aug 182009

First published online:

Aug 282009 
or development of extrapulmonary infection. Whether absolute PCT or CRP values on any day can predict survival is not known, since LuYT et al. [11] used a composite outcome that incorporated both survival and recurrent infection information.

SELIGMAN et al. [12] suggested that decreases in either serum PCT or CRP levels between onset and the fourth day of treatment could predict survival of VAP patients. However, the performance of this predictive rule has never been prospectively validated in a population that is different from the population in which it was originally derived [13].

We hypothesised that serum PCT and CRP levels on days 1 (D1), 4 (D4) and 7 (D7) of VAP can predict survival. We performed a study in which we evaluated: 1) serum PCT and CRP levels on the first, fourth and seventh days of VAP; and 2) CRP and PCT kinetics as predictors of the 28-day survival of VAP.

Elevated PCT concentrations have been suggested as promising indicators of sepsis and septic shock in critically ill patients [14]. However, the predictive performance of PCT or CRP for the development of septic shock in VAP patients has not been tested before. We also hypothesised that CRP and PCT levels or kinetics could serve as predictors for the development of septic shock in patients with VAP.

\section{MATERIALS AND METHODS \\ Study design and population}

The study was conducted at our ICU (Sotiria Chest Hospital, Athens, Greece), harbouring a population of mostly medical patients, over a 18-month period (April 2006 to September 2007). All patients consecutively admitted to the ICU suspected of VAP were eligible. The next of kin provided the informed consent for each patient included. The local ethics committee approved the study.

Initially, 54 patients with VAP were screened. Patients with community-acquired pneumonia as a cause of ICU hospitalisation $(n=3)$, patients with extrapulmonary infection $(n=1)$ as well as immunocompromised patients (haematological malignancies, HIV, neutropenia $<1,000$ cells $\cdot \mathrm{mL}^{-1}$, patients who had received chemotherapy within the preceding 45 days; $n=2$ ) were excluded from the study. Patients who died within the first 3 days after VAP diagnosis $(n=3)$ were also excluded from the study.

Finally, 45 patients $\geqslant 18$ yrs ( 34 male and 11 female, mean age \pm SD $61.5 \pm 17.8$ yrs) who developed VAP were enrolled in the study. VAP was defined as the occurrence of newly developed lung infiltrates, occurring $\geqslant 48 \mathrm{~h}$ after initiation of mechanical ventilation and persisting for $\geqslant 72 \mathrm{~h}$, plus two of the following three criteria: 1 ) fever $>38.2^{\circ} \mathrm{C}$; 2 ) leukocytosis $>12,000 \mathrm{~mm}^{-3}$; 3) purulent endotracheal secretions [15].

Additionally, microbiological documentation was necessary with the growth of $\geqslant 1 \times 10^{4}$ colony-forming units ( $\mathrm{cfu}$ ) $\cdot \mathrm{mL}^{-1}$ of a microorganism in bronchoalveolar lavage (BAL), or $\geqslant 1 \times 10^{3} \mathrm{cfu} \cdot \mathrm{mL}^{-1}$ in protected brush specimens, or $\geqslant 1 \times 10^{6} \mathrm{cfu} \cdot \mathrm{mL}^{-1}$ in endotracheal secretions and/or the isolation of a pathogen from blood cultures $[5,6,16,17]$. Data collected included admission diagnosis (table 1), past medical history and vital signs. In addition, the following were evaluated daily: clinical examination, presence or absence of organ dysfunction(s) and/or infection, temperature, white blood cell count, blood chemistry, arterial oxygen tension/ inspiratory oxygen fraction and chest radiographs.

Patients were evaluated daily for evidence of VAP. After the establishment of VAP diagnosis, all patients received empirical antibiotic treatment. The day of VAP clinical diagnosis was defined as D1 and was the same day that empirical antibiotic treatment was started. The following days were accordingly termed as D2, D3, etc. Only the first episode of VAP was evaluated in each patient. None of the patients had recurrent VAP or extrapulmonary infection during the first 10 days of VAP. VAP recurrence was defined as a new VAP episode, that is new clinical and radiological signs compatible with pneumonia, and included persistent infection (the same pathogen responsible for the first episode), relapse (the same pathogen as in the first episode but after the end of antibiotic therapy) and new infection (another pathogen, at any time).

CRP and PCT levels were measured on D1, D4 and D7 in all subjects included in the study. The Acute Physiology and Chronic Health Evaluation (APACHE) II and Sequential Organ Failure Assessment (SOFA) scores were used to assess disease severity $[18,19]$. The APACHE II score was calculated during the first $24 \mathrm{~h}$ of ICU admission. The SOFA score was evaluated on the same days with CRP and PCT measurements (D1, D4, D7). Patient progress was followed until the 28th day after the diagnosis of VAP, when they were considered survivors. Patients who died before D28 were categorised as nonsurvivors.

The evolution of CRP and PCT concentration throughout the course of VAP was analysed comparing survivors and nonsurvivors and also comparing those who developed septic shock or not. Septic shock was defined according to consensus definitions [20].

Blood samples were collected on D1, D4 and D7. The samples were centrifuged at $500 \times g$ for $10 \mathrm{~min}$ and the plasma was aliquoted and stored at $-70^{\circ} \mathrm{C}$ until analysed in a single batch. Circulating levels of CRP were measured using an immunoturbumetric method with a commercially available kit (Dade Behring, Deerfield, IL, USA). PCT was determined with chemiluscence (Liaison Brahms PCT; Dia Sorin S.P.A., Saluggia, Italy). Normal values were $<0.30 \mathrm{mg} \cdot \mathrm{dL}^{-1}$ for CRP and $0.1-0.5 \mathrm{ng} \cdot \mathrm{mL}^{-1}$ for PCT.

\section{Statistical analysis}

Values were expressed as mean $\pm \mathrm{SD}$ or as median (interquartile range 25-75th percentile) in case of a skewed distribution. Comparisons between groups were performed using the Mann-Whitney U-test. Categorical variables were compared with the Chi-squared test. Correlations were performed using Spearman's r-test.

Dichotomized $\Delta$ was calculated by the formula: $\Delta=$ D4-D1, D7$\mathrm{D} 4$ and D7-D1. Therefore $\quad \Delta \mathrm{PCT}_{4-1}=\mathrm{PCT}_{\mathrm{D} 4}-\mathrm{PCT}_{\mathrm{D} 1}$, $\Delta \mathrm{PCT}_{7-4}=\mathrm{PCT}_{\mathrm{D} 7}-\mathrm{PCT}_{\mathrm{D} 4}$, etc. $\Delta>0$ means increasing values and $\Delta \leqslant 0$ means decreasing values. $\triangle \mathrm{PCT}, \triangle \mathrm{CRP}$ and $\triangle \mathrm{SOFA}$ were categorised as increasing or unchanged/decreasing.

A univariate logistic regression analysis was used to define risk factors associated with VAP survival and septic shock development. A multivariate logistic regression analysis model was constructed with either VAP survival or septic shock 
TABLE 1 Characteristics of patients with ventilator-associated pneumonia

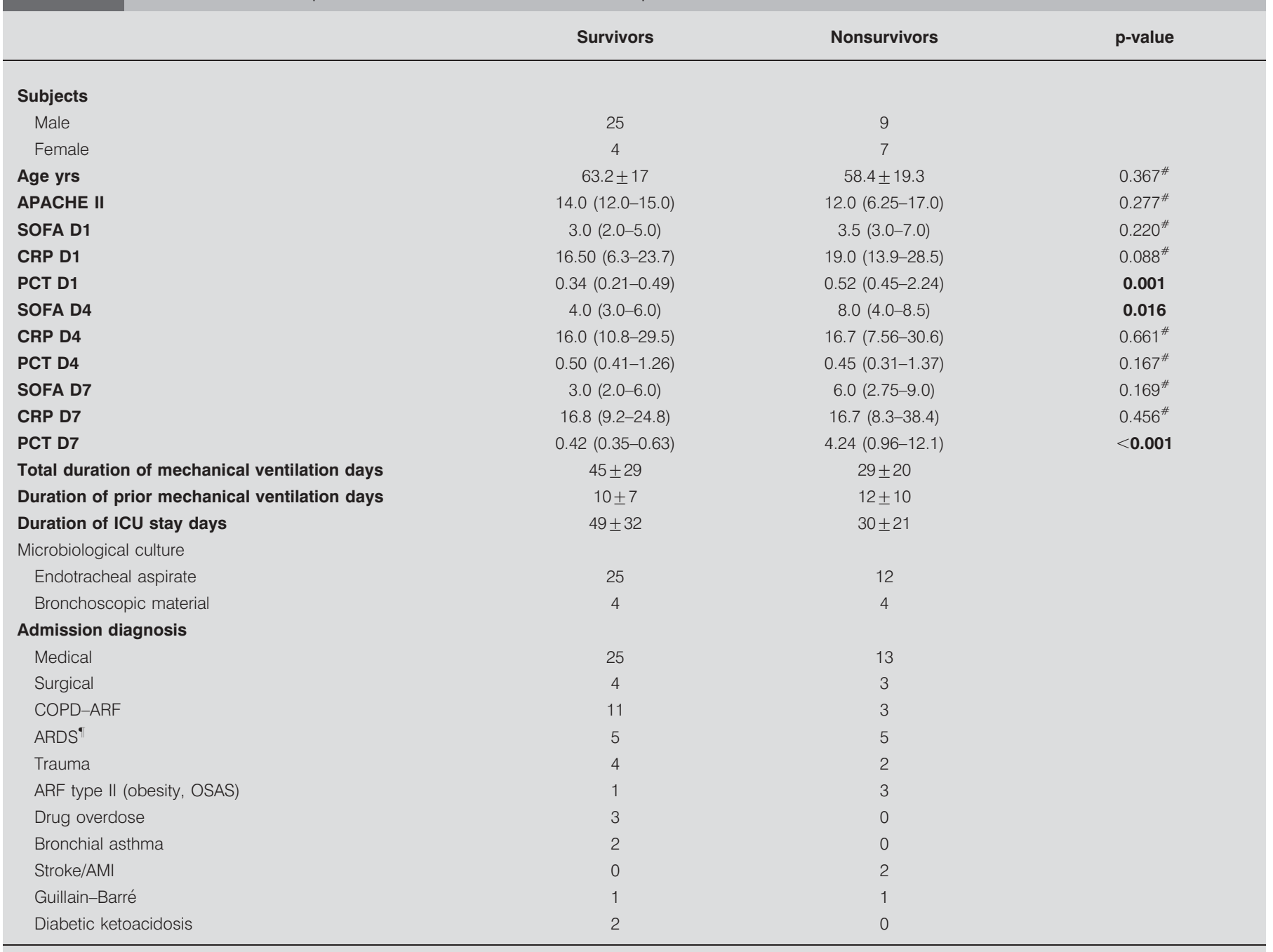

Data are presented as $\mathrm{n}$, mean $\pm \mathrm{SD}$ or median (interquartile range 25-75th percentile), unless stated otherwise. Values in bold are statistically significant. APACHE II: Acute Physiology and Chronic Health Evaluation II; SOFA: Sequential Organ Failure Assessment; CRP: C-reactive protein; PCT: procalcitonin; D: day; ICU: intensive care unit; COPD: chronic obstructive pulmonary disease; ARF: acute respiratory failure; ARDS: adult respiratory distress syndrome; OSAS: obstructive sleep apnoea syndrome; AMI: acute myocardial infarction. ${ }^{\#}$ : nonsignificant; " : acute pancreatitis $(n=3)$, multiple transfusions $(n=2)$, drug abuse $(n=2)$, near drowning $(n=1)$, inhalation of toxic gases $(n=1)$, lipid embolism $(n=1)$

development as the dependent variables, and CRP and PCT values on D1, D4 and D7, as well as variations in CRP and PCT $\left(\Delta \mathrm{CRP}_{4-1}, \Delta \mathrm{CRP}_{7-1}, \Delta \mathrm{CRP}_{7-4}, \Delta \mathrm{PCT}_{4-1}, \Delta \mathrm{PCT}_{7-1}, \Delta \mathrm{PCT}_{7-4}\right)$ as independent variables. To address potential collinearity, models were constructed that included only absolute values or only variations, as well as both absolute values and variations. To control for potential confounding factors, age, sex, APACHE II score and SOFA score were included in the initial model. Results are reported as adjusted OR with 95\% CI.

We prospectively validated the previously suggested CRP and PCT kinetics [12] by virtue of the design of our multivariate logistic regression analysis model. Receiver operating characteristic (ROC) curve analysis was performed for PCT and CRP values on the days that these variables differed between survivors and nonsurvivors and between VAP patients who did and did not develop septic shock.

Sensitivity, specificity, positive predictive value (PPV), negative predictive value (NPV), positive likelihood ratio (PLR; true-positive rate/false-positive rate or sensitivity/(1-specificity)) and negative likelihood ratio (NLR; false-negative rate/ true-negative rate or (1-sensitivity/specificity)) were calculated. Threshold values that gave the best combination of sensitivity and specificity were judged by calculating the Youden's index, i.e. the maximum difference between sensitivity and (1-specificity) [21].

The SPSS statistical package (version 14.0; SPSS, Chicago, IL, USA) was used. A p-value $<0.05$ was considered significant. 


\section{RESULTS}

During the study period, 45 patients developed VAP. The demographic characteristics, APACHE II and SOFA scores, and CRP and PCT values of patients are shown in table 1 .

In 26 out of 45 cases, a Gram-negative pathogen was isolated from the cultures of the lower respiratory tract; in one case only, a Gram-positive pathogen was isolated. Finally, in 18 cases, more than one pathogen was isolated. The more common pathogens isolated were Acinetobacter spp. in 14 cases and Pseudomonas aeruginosa in 10 cases. Klebsiella pneumoniae was isolated in two cases and Staphylococcus aureus in one case. Regarding the VAP cases that were polymicrobial, in 14 out of 18 cases two Gram-negative pathogens were isolated (again Acinetobacter spp. and P. aeruginosa were the most common) while a Gram-negative plus a Gram-positive were found in four cases.

In 37 patients the microbiological documentation of VAP was done by endotracheal aspirate cultures, while in eight patients samples obtained by fibreoptic bronchoscopy were used. Of the 45 patients studied, 16 (35.6\%) died before D28.

Positive blood cultures were obtained in 14 patients as follows: three Staphylococcus haemolyticus, two $S$. aureus, two Staphylococcus epidermidis, two P. aeruginosa, two K. pneumoniae, two Acinetobacter spp., one Enterococcus sp.

\section{VAP survival prediction}

\section{CRP and PCT levels}

CRP values exceeded the normal range in all patients with VAP on all measured days (D1, D4 and D7). No difference was found in CRP levels between survivors and nonsurvivors (table 1) However, nonsurvivors had significantly higher PCT levels on D1 and on D7 than survivors (table 1).

Areas under the curve (AUC) and 95\% CI for the prediction of survival for PCT on D1 and D7 were 0.793 (95\% CI 0.661-0.925) and 0.883 (95\% CI $0.770-0.995)$, respectively. Table 2 presents the best threshold values of PCT on D1 and D7, along with their predictive performance. The predictive performance of the PCT threshold values suggested by LUYT et al. [11] is also shown in table 2.

\begin{tabular}{|c|c|c|c|c|c|c|}
\hline TABLE 2 & $\begin{array}{l}\text { citoni } \\
\text { tor-as }\end{array}$ & $\begin{array}{l}\text { (PCT) } \\
\text { ociate }\end{array}$ & $\begin{array}{l}\text { resh } \\
\text { pneu }\end{array}$ & $\begin{array}{l}\text { d valu } \\
\text { onia }\end{array}$ & $\begin{array}{l}\text { and } \\
\text { tcom }\end{array}$ & \\
\hline РСТ & Sens. & Spec. & PPV & NPV & PLR & NLR \\
\hline D1 $0.42 \mathrm{ng} \cdot \mathrm{mL}^{-1}$ & 87.5 & 65.5 & 58.3 & 90.5 & 2.54 & 0.19 \\
\hline D7 $1.04 \mathrm{ng} \cdot \mathrm{mL}^{-1}$ & 80 & 86.2 & 66.6 & 92.6 & 5.78 & 0.23 \\
\hline $\mathrm{D} 1>1.0^{\#} \mathrm{ng} \cdot \mathrm{mL}^{-1}$ & 31 & 93 & 71 & 71 & 4.42 & 0.67 \\
\hline $\mathrm{D} 4>1.5^{\#} \mathrm{ng} \cdot \mathrm{mL}^{-1}$ & 25 & 79 & 33 & 72 & 1.19 & 0.69 \\
\hline $\mathrm{D} 7>0.5^{\#} \mathrm{ng} \cdot \mathrm{mL}^{-1}$ & 80 & 72 & 50 & 91 & 2.85 & 0.11 \\
\hline
\end{tabular}

Data are presented as \% unless otherwise stated. Sens.: sensitivity; Spec. specificity; PPV: positive predictive value; NPV: negative predictive value; PLR: positive likelihood ratio; NLR: negative likelihood ratio; D: day. ${ }^{*}$ : threshold values suggested by LuYT et al. [11].
In the multivariate analysis, neither $\mathrm{C}$-reactive protein nor PCT absolute values remained in the model predicting VAP survival.

\section{CRP and PCT kinetics}

In the univariate analysis, the factors associated with 28-day survival of VAP were the $\triangle \mathrm{PCT}_{7-1} \quad(\mathrm{p}=0.003), \Delta C R \mathrm{P}_{7-4}$ $(p=0.032), \Delta$ SOFA $_{4-1}(p=0.008)$ and the $\Delta$ SOFA $_{7-1}(p=0.037)$.

In the multivariate logistic regression analysis the factors remaining in the model were $\triangle \mathrm{PCT}_{7-1}(\mathrm{OR} 7.23,95 \% \mathrm{CI} 0.008$ $0.468 ; \mathrm{p}=0.007)$ and $\Delta \mathrm{CRP}_{7-4}(\mathrm{OR} 4.59,95 \%$ CI $0.013-0.824$; $\mathrm{p}=0.032)$.

\section{Septic shock development in VAP}

During the course of VAP, $22(48.9 \%)$ patients developed septic shock between the second and the fourth days (table 3). The SOFA score was significantly higher in patients with VAP who developed septic shock on all days (table 3). A positive correlation was detected between CRP levels and SOFA score on D1 $(\mathrm{r}=0.577, \mathrm{p}<0.001)$ and D7 $(\mathrm{r}=0.583, \mathrm{p}<0,001)$. Furthermore, positive correlations were found between PCT levels and SOFA score on D1 and PCT levels and CRP levels on $\mathrm{D} 1(\mathrm{r}=0.473, \mathrm{p}=0.001$ and $\mathrm{r}=0.352, \mathrm{p}=0.018$, respectively).

\section{CRP and PCT levels}

Serum CRP was higher in those who developed septic shock on D1 and on D7. Serum PCT was significantly higher on D1 and on D4 in those patients with VAP who developed septic

\begin{tabular}{|c|c|c|c|c|}
\hline \multirow[t]{2}{*}{ TABLE 3} & \multicolumn{3}{|c|}{$\begin{array}{l}\text { Septic shock development in ventilator- } \\
\text { associated pneumonia patients }\end{array}$} & \multirow[b]{2}{*}{ p-value } \\
\hline & & Nonseptic shock & Septic shock & \\
\hline Age yrs & & $64.1 \pm 16.1$ & $58.9 \pm 19.4$ & $0.569^{\#}$ \\
\hline Subjects & & 23 & 22 & \\
\hline Male & & 19 & 15 & \\
\hline Female & & 4 & 7 & \\
\hline Survivors & & 17 & 12 & \\
\hline Nonsurvivors & & 6 & 10 & \\
\hline APACHE II & & $13.0(7.0-15.0)$ & $13.0(10.5-16.5)$ & $0.340^{\#}$ \\
\hline SOFA D1 & & $3.0(2.0-3.0)$ & $6.5(3.0-8.0)$ & $<0.001$ \\
\hline CRP D1 & & $12.4(5.7-20.1)$ & $19.7(17.2-31.7)$ & 0.001 \\
\hline РCT D1 & & $0.37(0.22-0.48)$ & $0.55(0.30-2.46)$ & 0.001 \\
\hline SOFA D4 & & $3.0(2.0-4.0)$ & $8.0(6.0-9.0)$ & $<0.001$ \\
\hline CRP D4 & & $14.6(6.3-32.2)$ & $19.8(14.5-29.8)$ & $0.163^{\#}$ \\
\hline РCT D4 & & $0.41(0.32-0.47)$ & $0.56(0.49-1.67)$ & 0.005 \\
\hline SOFA D7 & & $2.0(2.0-3.0)$ & $8.0(6.0-9.25)$ & $<0.001$ \\
\hline CRP D7 & & $9.3(6.0-24.8)$ & $18.6(17.6-26.6)$ & 0.002 \\
\hline РСТ D7 & & $0.43(0.35-1.13)$ & $0.72(0.39-2.58)$ & $0.159^{\#}$ \\
\hline \multicolumn{5}{|c|}{ Microbiological culture } \\
\hline \multicolumn{2}{|c|}{ Endotracheal aspirate } & 20 & 17 & \\
\hline \multicolumn{2}{|c|}{ Bronchoscopic material } & 3 & 5 & \\
\hline
\end{tabular}

Data are presented as mean $\pm \mathrm{SD}, \mathrm{n}$ or median (interquartile range 25-75th percentile), unless stated otherwise. Values in bold are statistically significant. APACHE II: Acute Physiology and Chronic Health Evaluation II; SOFA: Sequential Organ Failure Assessment; D: day; CRP: C-reactive protein; PCT: procalcitonin. ${ }^{\#}$ : nonsignificant. 


\begin{tabular}{|c|c|c|c|c|c|c|}
\hline \multirow[t]{2}{*}{ TABLE 4} & \multicolumn{6}{|c|}{$\begin{array}{l}\text { Procalcitonin (PCT) and C-reactive protein (CRP) } \\
\text { threshold values and septic shock development }\end{array}$} \\
\hline & Sens. & Spec. & PPV & NPV & PLR & NLR \\
\hline $\begin{array}{l}\text { PCT D1 } \\
0.49 \mathrm{ng} \cdot \mathrm{mL}^{-1}\end{array}$ & 72.7 & 91.3 & 80 & 76 & 8.36 & 0.30 \\
\hline $\begin{array}{l}\text { CRP D1 } \\
15.2 \mathrm{ng} \cdot \mathrm{mL}^{-1}\end{array}$ & 86.4 & 65.2 & 70.4 & 83.3 & 2.48 & 0.21 \\
\hline $\begin{array}{l}\text { РCT D4 } \\
0.48 \mathrm{ng} \cdot \mathrm{mL}^{-1}\end{array}$ & 83.3 & 78.3 & 75 & 85.7 & 3.84 & 0.21 \\
\hline $\begin{array}{l}\text { CRP D7 } \\
\quad 15.75 \mathrm{ng} \cdot \mathrm{mL}^{-1}\end{array}$ & 93.8 & 73.9 & 71.4 & 94.4 & 3.59 & 0.08 \\
\hline
\end{tabular}

Data are presented as \% unless stated otherwise. Sens.: sensitivity; Spec specificity; PPV: positive predictive value; NPV: negative predictive value; PLR positive likelihood ratio; NLR: negative likelihood ratio.

shock (table 4). Additionally, among VAP patients with septic shock, nonsurvivors had significantly higher PCT levels on D1 and D7 than survivors (1.48 versus $0.43 \mathrm{ng} \cdot \mathrm{mL}^{-1}, \mathrm{p}=0.05$, and 7.16 versus $0.47 \mathrm{ng} \cdot \mathrm{mL}^{-1}, \mathrm{p}=0.04$, respectively).

AUC (95\% CI) for the prediction of septic shock development for PCT on D1 and D4 were $0.777(0.627-0.926)$ and 0.761 (0.597-0.925), respectively, and for CRP on D1 and D7 they were $0.794(0.664-0.925)$ and $0.783(0.626-0.939)$. The best threshold values of PCT and CRP along with their predictive performance on the respective days are presented in table 4 .

The only variable that remained in the model predicting the development of septic shock among VAP patients was SOFA on D1 (OR 7.44, 95\% CI 1.330-5.715; $\mathrm{p}=0.006)$. The AUC (95\% CI) for the prediction of septic shock development for SOFA on D1 was 0.830 (0.701-0.959). The best threshold value of SOFA score on D1 was 3.5 (sensitivity $72.7 \%$, specificity $82.6 \%$, PPV $80 \%$, NPV 76\%, PLR 4.18 and NLR 0.33).

\section{CRP and PCT kinetics}

In the univariate analysis, the factors associated with the development of septic shock were the $\Delta C R P_{7-4}(p=0.009)$ and the $\Delta \mathrm{SOFA}_{7-1}(\mathrm{p}=0.017)$. In the multivariate analysis, neither CRP nor PCT kinetics remained in the model predicting the development of septic shock.

\section{DISCUSSION}

To our knowledge, this is the first study to prospectively validate the performance of PCT and CRP kinetics, previously suggested in the literature as predictors of VAP survival, in a population that is different from the populations from which they were originally derived (derivation group). Such an approach is essential when the performance of a clinical prediction rule or diagnostic test is to be evaluated, since it shows whether the results initially obtained from one population (derivation group) are applicable to a different population and thus, could be generalised [13].

CRP and PCT threshold values as predictors of VAP survival Numerous studies have evaluated the usefulness of CRP [22, $23]$ and PCT [24, 25] both in the diagnosis and the prognosis of
VAP. PóvOA et al. [26] suggested that daily CRP measurements were useful in the identification, as early as D4, of VAP patients with poor outcome. In our study, serum CRP levels on D1, D4 and D7 during the course of VAP did not discriminate survivors from nonsurvivors.

LUYT et al. [11] have suggested that serum PCT levels on D1, D3 and D7 during the course of VAP are strong predictors of unfavourable outcome defined as either death, recurrent VAP or extrapulmonary infection. In our study, we focused on survival as our primary outcome. Although we found that serum PCT levels were significantly higher in nonsurvivors on D1 and D7 than in survivors, similar to previous studies [11, 27], and the AUC was satisfactory ( 0.79 on D1 and 0.88 on D7, suggesting moderate accuracy), the PLR was always $<10$ (2.54 on D1 and 5.78 on D7). A PLR of 10 is considered the threshold above which the PLR is considered really important [28, 29]. Furthermore, in the multivariate analysis, the PCT levels on either day were eliminated. However, it has to be acknowledged that the PLR of PCT on D7 was 5.78, which can generate moderate shifts in pre-test to post-test probability [28, 29].

\section{CRP and PCT kinetics as predictors of VAP survival}

SELIGMAN et al. [12] have suggested that decreasing CRP and PCT values between the onset and the 4th day of VAP could predict survival. In our group of VAP patients, CRP and PCT kinetics between D1, D4 and D7 were not able to predict survival.

The fact that CRP and PCT levels decreased on D7 compared with D1 in most survivors in our study lacks clinically useful prognostic significance. In fact, this result is rather expected, since 7 days after the initiation of antibiotic treatment, VAP has either responded to treatment and progresses towards resolution, or is refractory to it. Decreasing CRP and PCT levels between D1 and D4 would have contained clinically useful predictive information, but this could not be confirmed by our results.

\section{Prediction of septic shock development in VAP}

In this study we tested the hypothesis that CRP and PCT levels and kinetics could convey prognostic information for the development of septic shock in patients with VAP. We found that among patients with VAP, serum PCT was significantly higher on D1 and D4 in those who subsequently developed septic shock. Although the AUC suggested moderate accuracy $(0.75-0.78)$, the PLR was again $<10$ (table 4 ), implying that the results of the test are not likely to alter clinical decisions [28, 29]. Furthermore, in the multivariate analysis, PCT on D1 and D4 could not predict the development of septic shock. Again, the PLR of PCT on D1, 8.36, suggests the potential for useful predictive information.

The only predictor of septic shock development in our study was the SOFA score. Although the SOFA score was significantly higher in VAP patients with septic shock on all days (D1, D4, D7), only the SOFA score on D1 was predictive of septic shock development. To our knowledge, this is the first study showing that the SOFA score on the day that VAP is diagnosed can predict septic shock development. 


\section{Critique of methods: limitations}

Some limitations of our study should be noted. The number of patients included in the study is rather small, and thus our study was inadequately powered for the multivariate analysis performed. This is a rather common limitation with studies of this type in the field. The inadequate power increases the risk of underfitting (type II error), which could have led to the omission of important predictors from the model [30]. However, the performance of the same predictors in the univariate analysis of the ROC curves with the associated likelihood ratios of the best threshold values showed similar results, with moderate predictive performance at best and likelihood ratios always $<10$, which is the lower limit above which a test can generate large and often conclusive changes from pre-test to post-test probability [29].

Although we made every effort to exclude other causes of systemic infection, measured PCT levels cannot solely be attributed to pulmonary infection. The APACHE II score of our VAP patients was lower than that observed in other studies looking at outcomes. This could have some influence on our results. It should be kept in mind that the majority of our ICU patients are medical critically ill patients.

In our study, the diagnosis of VAP was mainly based upon quantitative endotracheal aspiration cultures, with a minority of patients diagnosed based on the cultures of bronchoscopically obtained material. Although invasive bronchoscopic strategies might be useful in permitting the de-escalation or cessation of unnecessary antimicrobial therapy [31], it is unlikely that the technique we used has significantly influenced our results, given that endotracheal aspirate cultures are associated with similar clinical outcomes when compared with quantitative cultures of BAL [32].

The incidence of septic shock among our VAP patients was relatively high $(48.9 \%)$. This may partly be attributed to the high number of multidrug-resistant pathogens as causative agents of VAP.

\section{Conclusions: implications}

Our findings cannot suggest the routine use of CRP and PCT levels as prognostic markers for the survival or septic shock development in patients with VAP. The PLRs of the PCT on D1 for septic shock development and D7 for survival suggest that the measurement of PCT can provide useful information. However, the context for using this information has to be investigated and our study was not designed to address this issue. For instance, serial serum PCT measurements have been suggested as indicators of the need to change treatment early in patients with $\mathrm{VAP}$, either to intensify treatment when PCT levels remain elevated, or to avoid unnecessary prolonged courses of antibiotics when levels are rapidly decreasing. More studies are needed to address the use of these markers in critically ill patients.

\section{STATEMENT OF INTEREST}

None declared.

\section{REFERENCES}

1 Rello J. Bench-to-bedside review: Therapeutic options and issues in the management of ventilator-associated bacterial pneumonia. Crit Care 2005; 9: 259-265.
2 Vincent JL, Bihari DJ, Suter PM, et al. The prevalence of nosocomial infection in intensive care units in Europe. Results of the European prevalence of infection in intensive care (EPIC) study. JAMA 1996; 274: 639-644.

3 Celis R, Torres A, Gatell JM, et al. Nosocomial pneumonia: a multivariate analysis of risk and prognosis. Chest 1988; 93: 318-324.

4 Fagon JY, Chastre J, Vuagnat A, et al. Nosocomial pneumonia and mortality among patients in intensive care units. JAMA 1996; 275: 866-869.

5 Ewig S, Bauer T, Torres A. The pulmonary physician in critical care 4: nosocomial pneumonia. Thorax 2002; 57: 366-371.

6 Chastre J, Fagon JY. Ventilator-associated pneumonia. Am J Respir Crit Care Med 2002; 165: 867-903.

7 Bowton DL. Nosocomial pneumonia in the ICU: year 2000 and beyond. Chest 1999; 115: 28-33.

8 Ueda S, Nishio K, Minamino N, et al. Increased plasma levels of adrenomedulin in patients with systemic inflammatory response syndrome. Am J Respir Crit Care Med 1999; 160: 132-136.

9 Boucher BA, Hanes SD. Searching for simple outcome markers in sepsis: an effort in futility? Crit Care Med 1999; 27: 1390-1391.

10 Becker KL, Nylén ES, White JC, et al. Procalcitonin and the calcitonin gene family of peptides in inflammation, infection and sepsis: a journey from calcitonin back to its precursors. J Clin Endocrinol Metab 2004; 89: 1512-1525.

11 Luyt CE, Guerin V, Combes A, et al. Procalcitonin kinetics as a prognostic marker of ventilator-associated pneumonia. Am J Respir Crit Care Med 2005; 171: 48-53.

12 Seligman R, Meisner M, Lisboa TC, et al. Decreases in procalcitonin and C-reactive protein are strong predictors of survival in ventilator-associated pneumonia. Crit Care 2006; 10: R125.

13 Laupacis A, Sekar N, Stiell IG. Clinical prediction rules. A review and suggested modifications on methodological standards. JAMA 1997; 277: 488-494.

14 Harbarth S, Holeckova K, Froidevaux C, et al. Diagnostic value of procalcitonin, interleukin-6, and interleukin-8 in critically ill patients admitted with suspected sepsis. Am J Respir Crit Care Med 2001; 164: 396-402.

15 Fabregas N, Ewig S, Torres A, et al. Clinical diagnosis of ventilator associated pneumonia revisited: comparative validation using immediate post-mortem lung biopsies. Thorax 1999; 54: 867-873.

16 Meduri GU, Chastre J. The standardization of bronchoscopic techniques for ventilator-associated pneumonia. Chest 1992; 102: 557-564.

17 Chastre J, Fagon JY, Bornet-Lesco M, et al. Evaluation of bronchoscopic techniques for the diagnosis of nosocomial pneumonia. Am J Respir Crit Care Med 1995; 152: 231-240.

18 Knaus WA, Draper EA, Wagner DP, et al. APACHE II: a severity of disease classification system. Crit Care Med 1985; 13: 818-829.

19 Vincent JL, de Mendonca A, Cantraine F, et al. Use of the SOFA score to assess the incidence of organ dysfunction/failure in intensive care units: results of a multicenter, prospective study. Working group on "sepsis-related problems" of the European Society of Intensive Care Medicine. Crit Care Med 1998; 26: 1793-1800.

20 Dellinger RP, Carlet JM, Masur H, et al. Surviving Sepsis Campaign guidelines for management of severe sepsis and septic shock. Intensive Care Med 2004; 30: 536-555.

21 Youden WJ. Index for rating diagnostic tests. Cancer 1950; 3: 32-35.

22 Povoa P, Coelho L, Almeida E, et al. C- reactive protein as a marker of infection in critically ill patients. Clin Microbiol Infect 2005; 11: 101-108.

23 Sierra R, Rello J, Bailen MA, et al. C-reactive protein used as an early indicator of infection in patients with systemic inflammatory response syndrome. Intensive Care Med 2004; 30: 2038-2045.

24 Ugarte H, Silva E, Mercan D, et al. Procalcitonin used as a marker of infection in the intensive care unit. Crit Care Med 1999; 27: 498-504. 
25 Luzzani A, Polati E, Dorizzi R, et al. Comparison of procalcitonin and C-reactive protein as markers of sepsis. Crit Care Med 2003; 31 1737-1741.

26 Póvoa P, Coelho L, Almeida E, et al. Early identification of intensive care unit-acquired infections with daily monitoring of Creactive protein: a prospective observational study. Crit Care 2006; 10: R63.

27 Duflo F, Debon R, Monneret G, et al. Alveolar and serum procalcitonin: diagnostic and prognostic value in ventilatorassociated pneumonia. Anesthesiology 2002; 96: 74-79.

28 Fischer JE, Bachmann LM, Jaeschke R. A readers' guide to the interpretation of diagnostic test properties: clinical example of sepsis. Intensive Care Med 2003; 29: 1043-1051.
29 Jaeschke R, Guyatt GH, Sackett DL. Users' guides to the medical literature. III. How to use an article about a diagnostic test. B. What are the results and will they help me in caring for my patients? The Evidence-Based Medicine Working Group. JAMA 1994; 271: 703-707.

30 Peduzzi P, Concato J, Kemper E, et al. A simulation study of the number of events per variable in logistic regression analysis. J Clin Epidemiol 1996; 49: 1373-1379.

31 Kollef MH. Diagnosis of ventilator-associated pneumonia. N Engl J Med 2006; 355: 2691-2693.

32 Ruiz M, Torres A, Ewig S, et al. Noninvasive versus invasive microbial investigation in ventilator-associated pneumonia: evaluation of outcome. Am J Respir Crit Care Med 2000; 162: 119-125. 